\title{
Increasing calculation accuracy in the design stage of a gas transportation system
}

\author{
Sorin NEACŞU and Renata RĂDULESCU*
}

Department of Drilling, Extraction and Transport of Hydrocarbons, Petroleum - Gas University of Ploiesti, Blvd. Bucharest, no.39, Ploiesti, Prahova County, Romania

\begin{abstract}
The paper presents a critical evaluation of the equations used to calculate the flow and pressure for transport pipelines. A highly accurate method for calculating gas parameters is proposed.

The final part of the paper presents some comparisons between the proposed method and modern simulators used for the design of transport systems.
\end{abstract}

Keywords. Gas, transport, capacity

\section{Introduction}

The transport capacity of a pipeline system dedicated to natural gas transportation is an important parameter. The transport capacity is related to the natural gas transported through the pipeline at a specific flow rate and under certain conditions. This transport capacity is sold to the interested customers by contracts.
Due to the variation of the consumption, usually the transport capacity is only partially covered by contracts. The difference between the firm capacity contracted and the technological capacity can also be sold as interruptible capacity. Figure 1 presents in a graphical form the different types of capacity defined in the literature [3-7].

Given the importance of the transport pipeline capacity, the paper presents a critical comparative analysis of calculation methods.

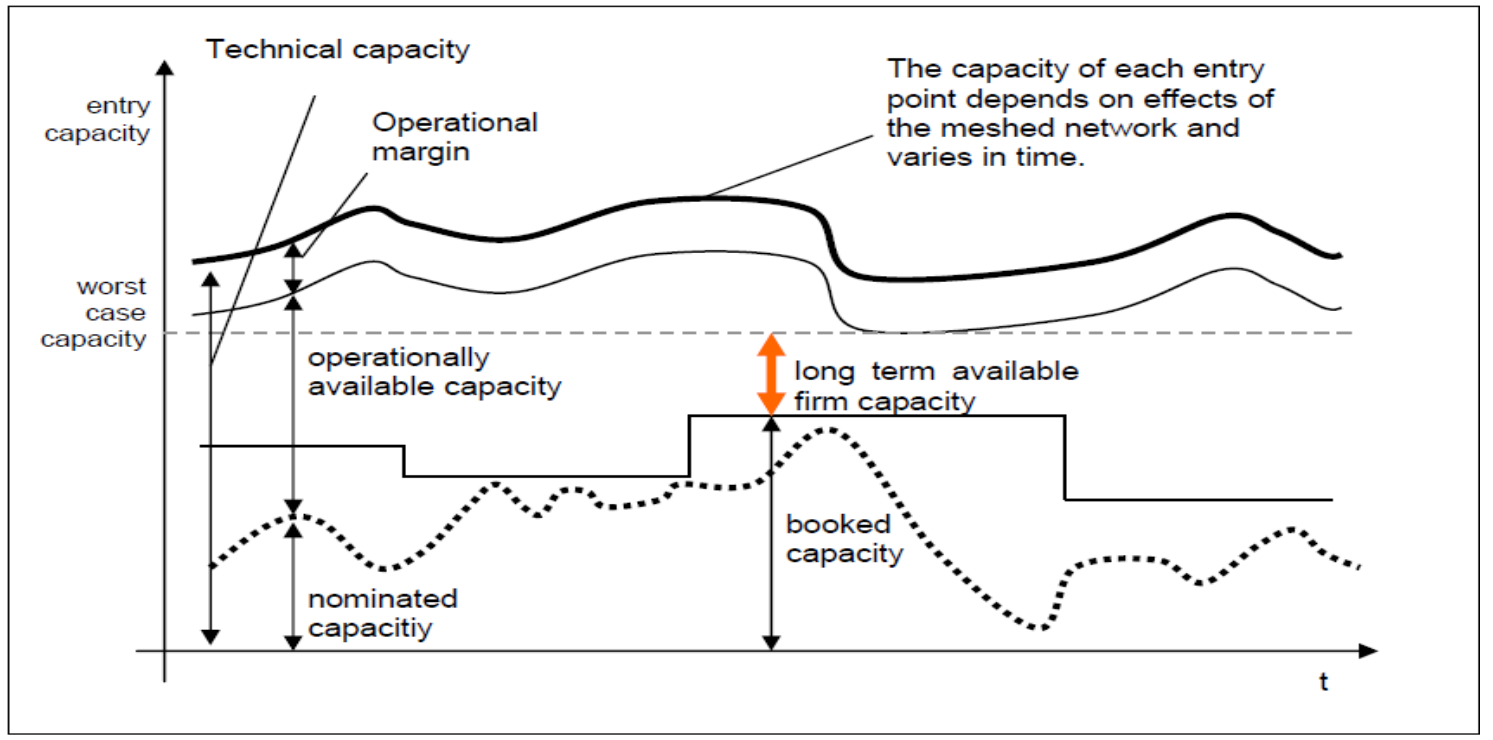

Fig. 1 Graphic representation of measures connected with transport capacity [7] 


\section{The classic calculation method for the capacity of a pipeline}

The momentum equation for one-dimensional steady movement of a gas in the pipeline is:

$$
w \frac{\mathrm{d} w}{\mathrm{~d} x}=-\frac{1}{\rho} \frac{\partial p}{\partial x}-\frac{w^{2}}{2} \frac{\lambda}{d}=0 .
$$

where $w$ is gas velocity

$$
\begin{aligned}
& p \text { - pressure } \\
& \rho \text { - density } \\
& \lambda \text { - hydraulic linear resistance } \\
& d \text { - pipeline diameter } \\
& x \text { - flowing direction }
\end{aligned}
$$

The continuity equation for one-dimensional steady movement is

$$
\frac{\partial}{\partial x}(\rho w)=0 \Rightarrow \rho w=\text { const }
$$

For a real gas, the state equation is

$$
\frac{p}{\rho}=Z(p, T) R T
$$

where $Z(p, T)$ is the compressibility factor from ideal gas law.

If we consider the movement of a gas through pipelines as isothermal at an average temperature $T$ and the compressibility factor $\mathrm{Z}$ to be constant along the pipeline, by integrating equation (1) according to GTE recommendations the result is the technical transport capacity of pipelines, the volume flow expressed at standard state that can be transported through a pipeline between two points, the input pressure $\mathrm{p}_{1}$ being the maximum operating pressure and the output pressure being defined as the minimum acceptable pressure due to technical or contractual reasons.

$$
Q_{n}=\frac{\pi}{4} \sqrt{\frac{\left(p_{1}^{2}-p_{2}^{2}\right) T_{n}}{p_{n} \rho_{n} l Z T}} \cdot \sqrt{\frac{d^{5}}{\lambda}}
$$

where $Q_{n}$ - gas flow in normal conditions, $\mathrm{St} \mathrm{m}^{3} \mathrm{~h}^{-1}$

$T_{n}-$ standard temperature, $288.15 \mathrm{~K}$

$p_{n}$ - normal pressure 1.01325 [bar]

$p_{1}$ - pipeline input pressure [bar]

$p_{2}$ - pipeline output pressure [bar]

$R$ - gas constant $\left[\mathrm{J} \cdot \mathrm{kg}^{-1} \cdot \mathrm{K}^{-1}\right]$

$T$ - absolute gas temperature $(T=t+273.15 ; t-$ temperature in ${ }^{\circ} \mathrm{C}$ ) $[\mathrm{K}]$

$l$ - pipeline length $[\mathrm{m}]$

\section{Calculation method for the pipeline capacity with an improved accuracy, PGU method}

To increase the calculation accuracy of the pipeline capacity the simplifying assumptions necessary for the analytical integration of Bernoulli's equation are dropped and a numerical integration method based on a Runge Kutta method of order IV is used.

Momentum equation (1) can be integrated to obtain an analytical solution using simplifying assumptions, such as:

- The inertial term $w \frac{\mathrm{d} w}{\mathrm{~d} x}$ is neglected

- Gas density and compressibility factor are considered to vary along the pipeline.

In such conditions equation (1) becomes:

$$
\frac{\mathrm{d} p}{\mathrm{~d} x}=-\frac{\lambda}{2 d} \rho \mathrm{w}^{2} .
$$

For defining the integration scheme we consider the pipeline divided into segments of equal length (h), as shown in Figure 2.

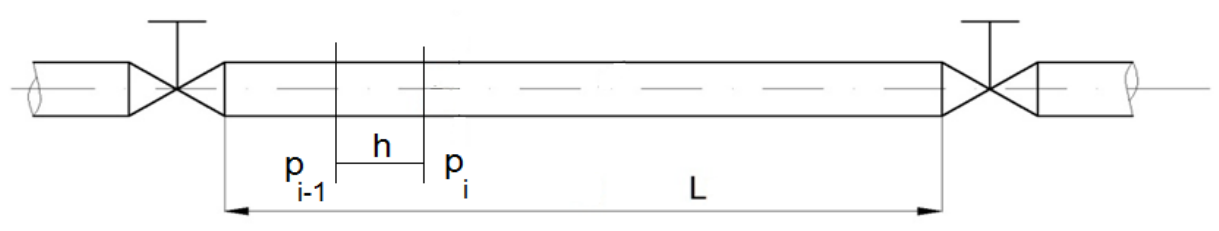

Fig. 2 Defining the numerical scheme is done for point $i$ using information from the previous poiny $i-1$ 
Starting from equation (5) a function depending on the pressure in the network nodes defined in the pipeline is expressed as follows:

$$
f(p)=-\lambda \rho \frac{\mathrm{w}^{2}}{2 d}
$$

(6)Based on it the terms corresponding to Runge Kutta method order IV are defined:

$$
\begin{aligned}
& k_{1}=f\left(p_{i-1}\right) \\
& k_{2}=f\left(p_{i-1}+0,5 \cdot h \cdot k_{1}\right) \\
& k_{3}=f\left(p_{i-1}+0,5 \cdot h \cdot k_{2}\right) \\
& k_{4}=f\left(p_{i-1}+0,5 \cdot h \cdot k_{3}\right) \\
& p_{i}=p_{i-1}+\frac{h\left(k_{1}+2 k_{2}+2 k_{3}+k_{4}\right)}{6}
\end{aligned}
$$

Equation (8) allows determination of the pressure variation along the pipeline at each point of the network nodes.

\section{The calculation method used as reference}

The most accurate methods are progressive methods according to which the pipeline is divided into segments and the equations governing the flow of each piece of the pipeline are solved. Progressive methods can be divided into two categories: variable length of the point and point length depending on Mach number.

Another approach is to resolve each pipeline as a whole by a single calculation. These methods are much faster than the progressive methods. The compromise decreases the accuracy and flexibility of the model. Inside the pipelines two processes are considered: adiabatic process for short pipelines and isothermal process for long ones.

For isothermal flow it is considered that there is an intensive exchange of heat between the gas and the external environment that leads to heat loss resulting from friction, so that the gas temperature remains constant. The equations which represent the base of the model are:

- Continuity equation (9)

$$
\frac{\mathrm{d} \rho}{\rho}+\frac{\mathrm{d} w}{w}=0
$$

where: $w$ - velocity, $\rho$-density.

- The momentum equation for unidimensional movement applied on pipelines with constant area is:

$$
\mathrm{d} p+\frac{\rho w^{2}}{2} \frac{f \mathrm{~d} w}{d}+\rho w \mathrm{~d} w+\rho g \mathrm{~d} z=0
$$

Where $f$ represents the friction factor and $z$ the altitude.

- State equation

$$
p=Z \rho R T
$$

The adapted point method based on Mach number

\section{Calculating the integration point}

By successive processing of equations (9), (10) and (11) the following equation results:

$$
\begin{aligned}
& \frac{\mathrm{d} M^{2}}{M^{2}}-F_{T_{0}}\left(\frac{\mathrm{d} T_{0}}{T_{0}}+\frac{\mathrm{d} Z}{Z}\right)-F_{k}\left(\frac{\mathrm{d} k}{k}\right)- \\
& F_{f} \frac{f \mathrm{~d} x}{D}-F_{g} \frac{g \sin \theta \mathrm{d} x}{Z R T_{0}}=0
\end{aligned}
$$

Where $M$ is the Mach number and the following notations were used:

$$
\left\{\begin{array}{l}
F_{T_{0}}=\frac{\left(k M^{2}+1\right)\left(1+\frac{k-1}{2} M^{2}\right)}{\left(1-M^{2}\right)} \\
F_{k}=\frac{\left(1-k M^{2}\right)\left(1+\frac{k-1}{2} M^{2}\right)}{\left(1-M^{2}\right)} \\
F_{f}=\frac{k M^{2}\left(1+\frac{k-1}{2} M^{2}\right)}{\left(1-M^{2}\right)} \\
F_{g}=1+\frac{k-1}{2} M^{2}
\end{array}\right.
$$

If a short segment of pipeline is considered gas parameters can be thought as constant along this segment. Under these conditions equation (13), which in this case is a differential equation of separable variables, can be integrated term by term, having the form (14): 


$$
\begin{aligned}
& \ln \frac{M_{2}^{2}}{M_{1}^{2}}-\bar{F}_{T_{0}}\left(\ln \frac{T_{02}}{T_{01}}+\ln \frac{Z_{2}}{Z_{1}}\right)-\bar{F}_{k}\left(\ln \frac{k_{2}}{k_{1}}\right)- \\
& \left(x_{2}-x_{1}\right)\left(\bar{F}_{f} \frac{f}{D}-\bar{F}_{g} \frac{g \sin \theta}{Z R T_{0}}\right)=0
\end{aligned}
$$

Equation (14) can be solved for $x_{2}$, this resulting the length of the segment for a low variation of Mach number from $\mathrm{M}_{1}$ to $\mathrm{M}_{2}$.

$$
x_{2}=x_{1}+\frac{\ln \frac{M_{2}^{2}}{M_{1}^{2}}-\bar{F}_{T_{0}}\left(\ln \frac{T_{02}}{T_{01}}+\ln \frac{Z_{2}}{Z_{1}}\right)-\bar{F}_{k}\left(\ln \frac{k_{2}}{k_{1}}\right)}{\left(\bar{F}_{f} \frac{f}{D}-\bar{F}_{g} \frac{g \sin \theta}{Z R T_{0}}\right)}(15)
$$

In equation (15) the barred parameters represent average values on the considered interval. Equation (15) is the connection between the calculated segment and Mach number. The calculation pitch of the pipeline varies depending on the Mach number.

$$
\begin{aligned}
& \ln \frac{p_{02}}{p_{01}}=-\frac{k \bar{M}^{2}}{2} \ln \frac{T_{02}}{T_{01}}-\frac{k \bar{M}^{2}}{2} \ln \frac{Z_{2}}{Z_{1}}-\frac{k \bar{M}^{2}}{2} . \\
& \cdot \ln \frac{k_{2}}{k_{1}}-\frac{k \bar{M}^{2}}{2} \frac{f\left(x_{2}-x_{1}\right)}{D}-\frac{k \bar{M}^{2}}{2} \frac{n \theta\left(x_{2}-x_{1}\right)}{Z R T}
\end{aligned}
$$

Thermodynamic parameters of the flow are obtained depending on the pressure variation along the pipeline resulting from the integration of equation (16) in a basic section of the pipeline whose length is equal to $\mathrm{x}_{2}-\mathrm{x}_{1}$, value determined from equation (15). The solution of the equation (16) is:

$$
\begin{aligned}
& \left\{\begin{array}{l}
p_{02}=p_{01} \cdot e^{-C} \\
C=-\frac{k \bar{M}^{2}}{2}\left(\ln \frac{T_{02}}{T_{01}}+\ln \frac{Z_{2}}{Z_{1}}+\ln \frac{k_{2}}{k_{1}}+{ }_{(17)}\right.
\end{array}\right. \\
& \left.+\frac{f\left(x_{2}-x_{1}\right)}{D}+\frac{g \sin \theta\left(x_{2}-x_{1}\right)}{Z R T}\right)
\end{aligned}
$$

\section{Results and discussions}

Using the three methods previously presented, flow parameters were calculated along a 20 inch steel pipeline which is $30 \mathrm{~km}$ long. The transport process was considered as being isothermal at a temperature of $10^{\circ} \mathrm{C}$.

In order to compare the results in all three analyzed cases it was considered that the pipeline transported the same flow of 210,000 St $\mathrm{m}^{3} \mathrm{~h}^{-1}$. Values of the results are presented in graphical form.

Figure 3 presents a comparison between the classic and reference methods used to calculate the variation of gas pressure and velocity along the pipeline.

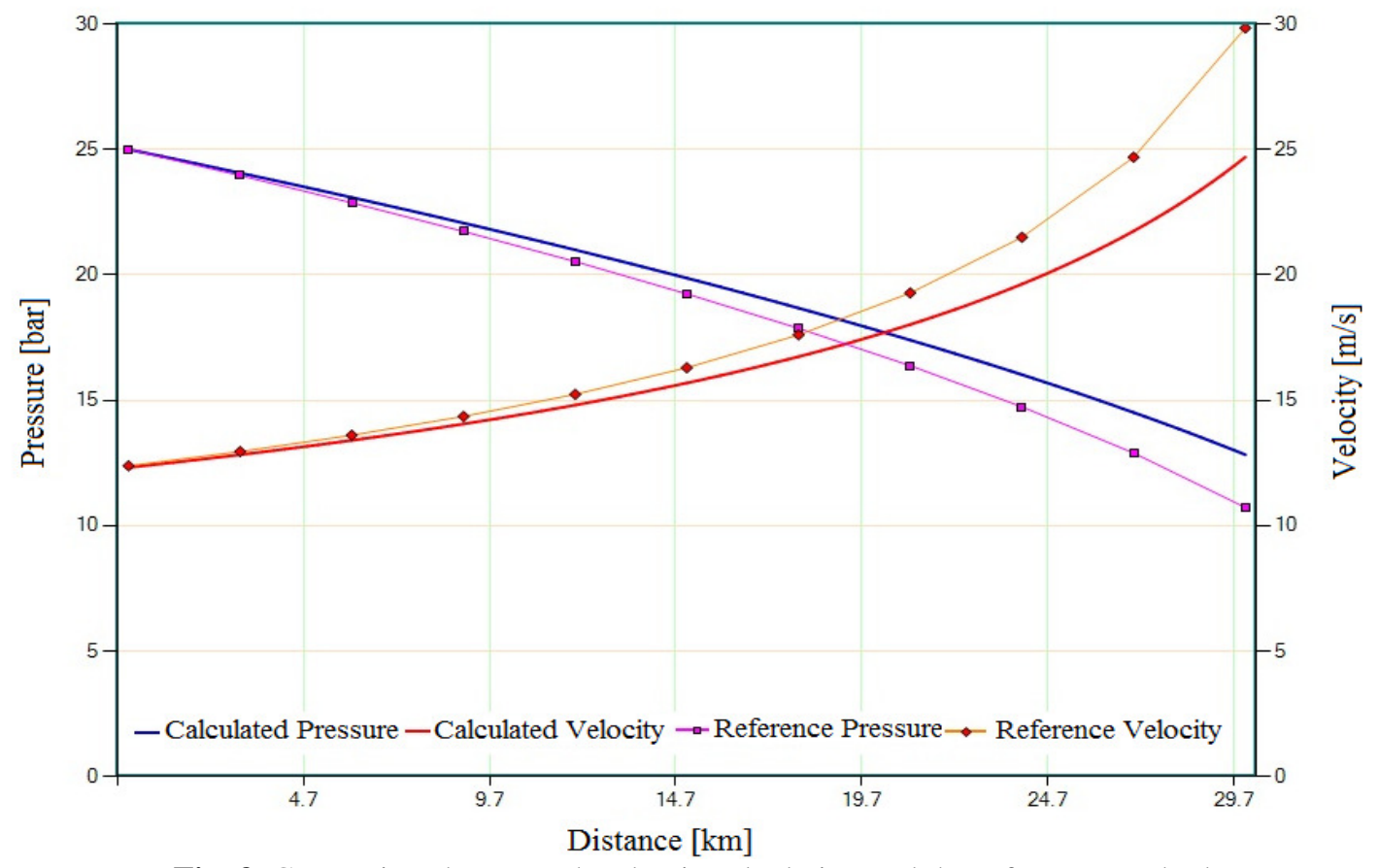

Fig. 3. Comparison between the classic calculation and the reference method 
This allows us to conclude that the reference method has better accuracy than the classical method, the calculated values being very close to the values obtained from the reference method.

Figure 4 shows the results of calculations made by using the PGU method compared to the reference method. It is noted that the values obtained from the two methods are close for the entire length of the pipeline. There is a substantial difference between the two methods. Analyzing the graphical values one can notice that the flow rates (pipeline capacity), which are determined by classic formulas, are lower in reality than the ones resulted from the calculations.

Figure 5 presents the relative errors for the calculation of gas pressure drop along the pipe by using the classical method and the PGU method.

It is easy to see that the errors of the PGU method

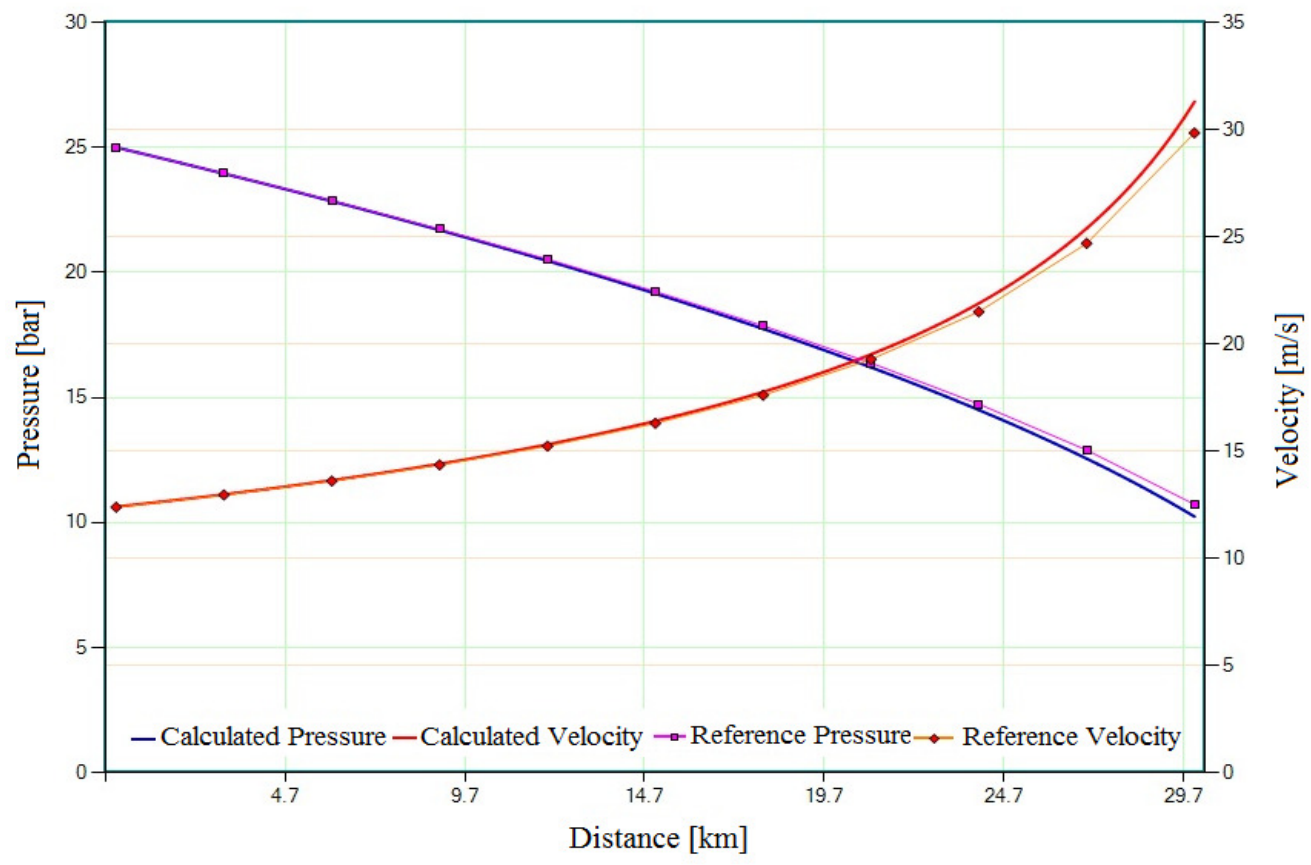

Fig. 4 Comparison between the PGU and the reference method

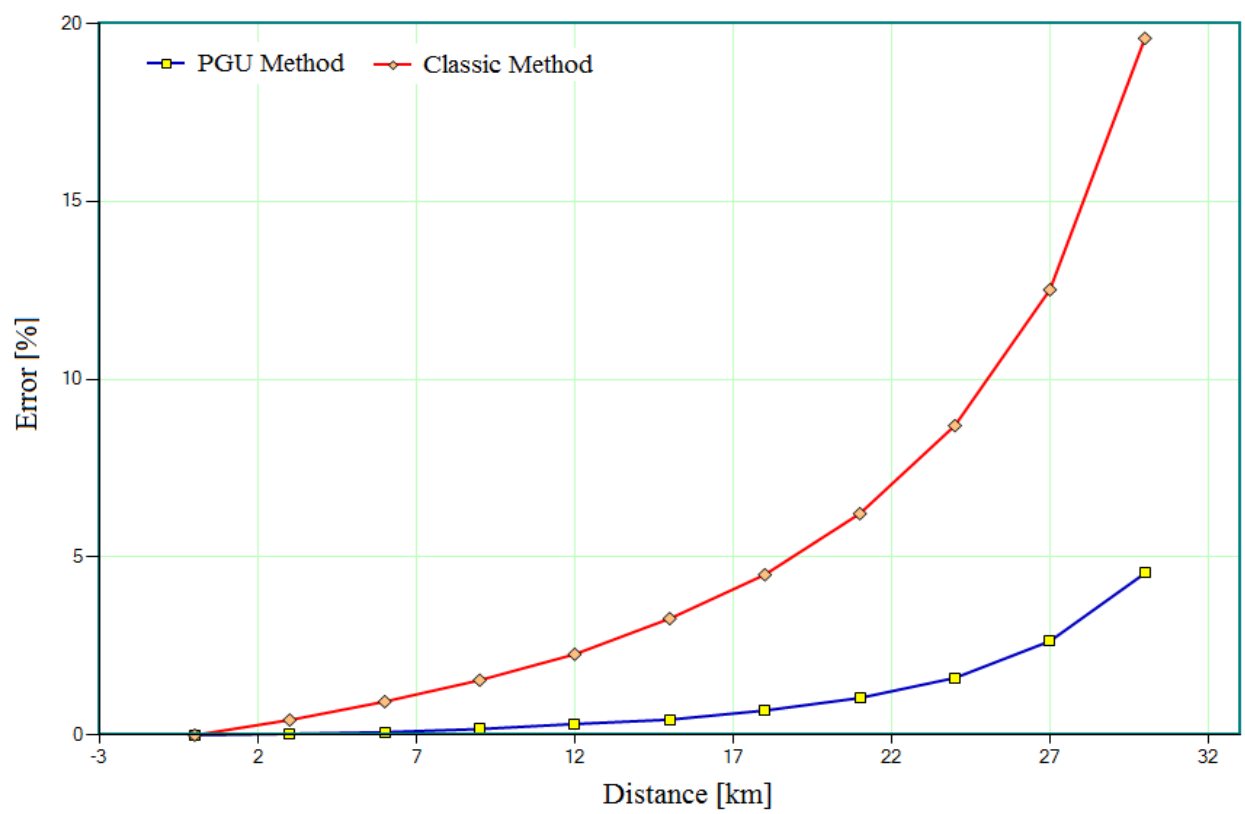

Fig. 5 Relative errors for the calculation of gas pressure drop along the pipe 
are maintained below $5 \%$, while the ones of the classic method reach $20 \%$ at the end of the pipe.

\section{Conclusions}

The most used method of calculating the flow rate for gas transportation pipelines is presented in the paper as the classic method. Comparative calculations with more accurate methods showed that the values obtained with the classic method are less accurate.

Given the importance of transport capacity value, this should be calculated using methods that provide high precision.

\section{References}

* E-mail address: renataradulescu@yahoo.com [1] M. Albulescu, S. Neacşu, C. Eparu, S. Bambo and S. Suditu, Termotehnica Revue, Supplement no. 1 (2011)
[2] http://www.aft.com/

[3] *** ERGEG - Capacity Allocation on European Gas Transmission Networks Pilot Framework Guideline, 10 June 2010;

[4] *** GTE - Capacity Product Coordination First Phase Report, 27 March 2009;

[5] *** GTE comments on - Calculation of Available Capacities: Understanding and Issues An ERGEG Public Consultation Paper, 09 August 2007;

[6] *** GTE position - Definition of available capacities at interconnection points in liberalized markets, 22 June 2004;

[7] *** REGULATION (EC) No 1775/2005 OF THE EUROPEAN PARLIAMENT AND OF THE COUNCIL of 28 September 2005 on conditions for access to the natural gas transmission networks (Text with EEA relevance)

Submitted: May $20^{\text {th }} 2013$ Accepted in revised form: August $5^{\text {th }} 2013$ 